\title{
Impacto dos Modos de Estimulação DDD e VIR na Capacidade Funcional e Qualidade de Vida de Pacientes Chagásicos
}

\author{
Impact of DDD and VIR Stimulation Modes on Functional Capacity and \\ Quality of Life of Chagasic Patients
}

\begin{abstract}
Débora Rodrigues Santana1, Geraldo Paulino Santana Filho1, Zander Bastos Rocha', Antonio Malan Cavalcanti Lima², Max Weyler Nery³, Salvador Rassi ${ }^{4}$, Giulliano Gardenghi,*
\end{abstract}

ORCID IDS

Santana DR (D) https://orcid.org/0000-0003-0659-8755

Santana Filho GP (D) https://orcid.org/0000-0002-3224-4435

Rocha ZB (D) https://orcid.org/0000-0002-1413-753X

Lima AMC (D) https://orcid.org/0000-0002-7252-9056

\section{RESUMO}

Introdução: A estimulação atrioventricular propicia benefícios hemodinâmicos em relação à ventricular isolada, mas essa vantagem não está completamente estabelecida em pacientes chagásicos com disfunção sistólica. Objetivo: Avaliar a influência dos modos de estimulação DDD e VVIR na capacidade funcional, qualidade de vida (QV) e alterações laboratoriais de peptídeo natriurético em pacientes chagásicos com disfunção ventricular submetidos a implante de marcapasso. Métodos: Estudaram-se prospectivamente 20 pacientes (55\% do sexo masculino) com média de idade de 62,7 ( \pm 9,9 anos) e média da fração de ejeção de $41,8 \%$ ( $\pm 2,8)$. Alternadamente, os pacientes receberam a estimulação nos modos DDD e VVIR por um período de três meses sob cada programação. O mínimo percentual de estimulação ventricular admitido foi de $80 \%$. Após cada período, o paciente foi submetido ao teste de caminhada de seis minutos (TC6M), avaliação de QV pelo Minnesota Living with Heart Failure Questionnaire (MLHFQ) e pelo Assesment of QUAlity of life and RELated events (AQUAREL). A avaliação laboratorial foi realizada com a dosagem da fração N-terminal do peptídeo natriurético cerebral (N-terminal pro b-type natriuretic peptide - NT-proBNP). Resultados: A média da distância percorrida no TC6M nos modos DDD e WIR foram respectivamente $390,60( \pm 52,71)$ e $396,30( \pm 52,71)$ metros $(p=0,160)$. Verificaram-se resultados de $Q V$ inferiores, considerando o domínio físico do MLHFQ ( $p=0,03)$ e os domínios dispneia de esforço $(p=0,05)$ e arritmia $(p<0,001)$ do AQUAREL, com o modo WIR. Os níveis de NT-proBNP aumentaram significativamente com a estimulação no modo VVIR ( $p<0,001)$. Conclusão: Após três meses de estimulação com o modo VVIR, houve piora da QV dos pacientes chagásicos e aumento dos níveis de NT-proBNP (registro de ensaio clínico: ReBEc RBR-53×476).

PALAVRAS-CHAVE: Doença de Chagas; Marcapasso; Qualidade de vida.
Nery MW (D) https://orcid.org/0000-0002-2944-6220

Rassi S (iD https://orcid.org/0000-0001-7210-6486

Gardenghi G (DD https://orcid.org/0000-0002-8763-561X

\begin{abstract}
Introduction: Atrioventricular stimulation provides hemodynamic benefits over the isolated ventricular rate, but this advantage is not completely established in chagasic patients with systolic dysfunction. Objectives: To evaluate the influence of DDD and VVIR stimulation modes on functional capacity, quality of life (QoL) and laboratory abnormalities of a natriuretic peptide in chagasic patients with ventricular dysfunction submitted to pacemaker implantation.Methods: Twenty patients (55\% male) with a mean age of 62.7 ( \pm 9.9 years) and a mean ejection fraction of $41.8 \%$ ( \pm 2.8 ) were prospectively studied. Alternately, patients received pacing in the DDD and VVIR modes for a period of three months under each schedule. The minimum percentage of ventricular pacing was $80 \%$. After each period, the patient was submitted to the six-minute walk test (6MWT), QOL assessment by the Minnesota Living with Heart Failure Questionnaire (MLHFQ) and the Assay of QUAlity of life and RELated events (AQUAREL). Laboratory evaluation was performed with the $\mathrm{N}$-terminal fraction of the brain natriuretic peptide ( $\mathrm{N}$-terminal pro b-type natriuretic peptide - NT-proBNP). Results: The mean distance walked on the 6MWT in the DDD and VVIR modes were $390.60( \pm 52.71)$ and $396.30( \pm 52.71)$ meters respectively $(p=0.160)$. Results of lower QOL were found, considering the physical domain of the MLHFQ $(p=0.03)$ and the domains of effort dyspnea $(p=0.05)$ and arrhythmia $(p<0.001)$ of the AQUAREL with the WIR mode. NT-proBNP levels increased significantly with stimulation in VVIR mode $(p<0.001)$. Conclusion: After three months of stimulation with the VVIR mode, there was worsening of the QoL of the chagasic patients and increase of the levels of NT-proBNP (clinical trial record: ReBEc RBR-53×476)
\end{abstract}

KEYWORDS: Chagas disease; Pacemaker; Quality of life.

1.Santa Casa de Misericórdia de Goiânia - Goiânia/GO - Brasil.

2.Pontifícia Universidade de Goiás - Goiânia/GO - Brasil.

3. Hospital ENCORE - Aparecida de Goiânia/GO - Brasil.

4. Universidade Federal de Goiás - Hospital das Clínicas - Serviço de Cardiologia - Goiânia/GO - Brasil.

*Autor correspondente: ggardenghi@encore.com.br

Recebido: 03 Jul 2018 | Aceito: 04 Fev 2019

Editor Associado: J. Tarcísio Medeiros de Vasconcelos 


\section{INTRODUÇÃO}

Apesar do declínio no número de novos casos de doença de Chagas (DCh), sua condição endêmica e cronicidade a sustentam como importante problema de saúde na América do $\mathrm{Sul}^{1}$. Dados recentes demonstram que 20 a $30 \%$ dos milhões de soropositivos manifestam, a cada ano, alguma forma de miocardiopatia ${ }^{2,3}$. O acometimento do sistema excito-condutor cardíaco pelo Trypanosoma cruzi permanece como segunda etiologia mais frequentemente envolvida no implante de marcapasso convencional no Brasil ${ }^{4}$. A importante redução de mortalidade nessa população consagrou a estimulação artificial como tratamento de escolha das bradicardias ${ }^{5}$.

A evolução estrutural e eletrônica dos marcapassos despertou o interesse para detecção de benefícios adicionais como a qualidade de vida (QV). A estimulação atrioventricular $(\mathrm{AV})$ vem se revelando superior em relação à estimulação unicameral que, por isso, tem sido preterida caso a atividade sinusal esteja presente ${ }^{6}$. Porém, as evidências quanto à $\mathrm{QV}$ e capacidade funcional têm como base populações sem DCh e com fração de ejeção preservada. Poucas informações correlacionam os resultados de QV e capacidade funcional com o emprego de ferramentas específicas para pacientes chagásicos com marcapasso.

\section{OBJETIVO}

Avaliar os efeitos dos modos de estimulação cardíaca artificial na $\mathrm{QV}$ e na capacidade funcional de pacientes chagásicos com bloqueio AV total submetidos a implante de marcapasso e detectar sua influência nos níveis da fração $\mathrm{N}$-terminal do peptídeo natriurético cerebral ( $N$-terminal pro b-type natriuretic peptide - NT-proBNP).

\section{MÉTODOS}

Trata-se de estudo clínico controlado, prospectivo, realizado na Santa Casa de Misericórdia de Goiânia (SCMG), estado de Goiás, em pacientes submetidos a implante de marcapasso dupla câmara, no período de julho de 2014 a abril de 2015. Após a aprovação do estudo pelo Comitê de Ética da SCMG (CAAE: 32745114.4.0000,5081), os pacientes foram avaliados na consulta de retorno que ocorre, em média, 10 dias pósimplante do marcapasso.

Fizeram parte dos critérios de inclusão a confirmação sorológica de $\mathrm{DCh}$, a comprovação do bloqueio AV total por eletrocardiograma ou holter prévio ao implante e fração de ejeção do ventrículo esquerdo inferior a 50\% pelo método de Simpson. A detecção de fibrilação atrial a qualquer tempo do seguimento, doença pulmonar obstrutiva crônica clinicamente importante, doença arterial coronária (com diagnóstico prévio ou angina de peito), presença de condição limitante da deambulação, insuficiência cardíaca classe funcional (CF) IV da New York Heart Association (NYHA) e estimulação ventricular artificial inferior a $80 \%$ foram considerados critérios de exclusão.

Os pacientes elegíveis foram alocados por ordem cronológica do implante em grupos A ou B para evitar o efeito de aprendizagem nas avaliações. Os participantes do grupo A tiveram seu modo de estimulação inicialmente programado em DDD e aqueles do grupo B em modo VVIR. Após 90 dias, foram submetidos à primeira avaliação que consistiu de: aplicação dos questionários de QV; realização do teste de caminhada de seis minutos (TC6M); e coleta de amostra de sangue para dosagem de NT-proBNP. Em seguida, os pacientes foram submetidos à reprogramação para o outro modo de estimulação, de acordo com o grupo ao qual pertenciam. Após 90 dias, a segunda avaliação foi realizada e os pacientes foram encaminhados ao ambulatório de marcapasso para decisão final da equipe assistente quanto ao modo de estimulação definitivo.

O TC6M foi utilizado como instrumento de avaliação funcional e realizado de acordo com as recomendações da American Thoracic Society (ATS) ${ }^{7}$. Aplicaram-se, em ambas as avaliações previstas, dois questionários: o Minnesota Living with Heart Failure Questionnaire (MLHFQ) e o Assesment of QUAlity of life and RELated events (AQUAREL).

O MLHFQ foi utilizado na versão transcultural publicada e validada em português, a qual engloba os domínios emocional e físico em 21 questões que, somadas, resultam no escore total em que valores maiores correspondem a melhor aspecto de $\mathrm{QV}^{8}$. O questionário AQUAREL é composto por 20 perguntas diretamente ligadas a aspectos relevantes aos pacientes com marcapasso, distribuídas em três domínios: desconforto no peito; dispneia de esforço; e arritmia. O NT-proBNP foi dosado pelo teste $\mathrm{Cardiac}^{\circledR}$ NT-proBNP $\left(\right.$ Roche $\left.^{\circledR}\right)$. 
No modo VVIR, a frequência programada foi de $60 \mathrm{bpm}$ e, como os pacientes apresentavam bloqueio AV de grau avançado, não houve, em nenhum momento, o sincronismo AV. Os dispositivos do modelo ADAPTAADDR03 (Medtronic Inc., Minneapolis, USA) contavam com um sensor do tipo acelerômetro e marcapassos ENTOVIS DR-T (Biotronik SE \& Co, Berlin, Germany) funcionaram com sensor de malha fechada. Para ambos os sensores, estabeleceu-se a frequência mínima de 60 e máxima de 130 bpm.

No modo DDD, a frequência mínima de estimulação foi de 50 bpm e a frequência máxima de $80 \%$ da máxima para a idade. A frequência máxima de seguimento (uppertrack rate) estabelecida foi de $130 \mathrm{bpm}$.

$\mathrm{O}$ intervalo $\mathrm{AV}$ não foi individualizado, sendo mantido o nominal de 120 ms após uma onda "p" espontânea e 150 ms após uma onda "p" estimulada. A ocorrência de intervalo AV dinâmico foi permitida.

\section{Análise Estatística}

A verificação de normalidade dos dados quantitativos foi realizada com o teste de Kolmogorov-Smirnov. As variáveis paramétricas foram analisadas pelo teste $t$ pareado. O teste de Wilcoxon foi aplicado para as variáveis não paramétricas. Com relação às frequências encontradas na classificação NYHA, utilizou-se o teste do quiquadrado post hoc, conforme descrito por Beasley et al. ${ }^{9}$ Para todas análises, adotou-se o nível de significância de $5 \%(p<0.05)$ com intervalo de confiança de 95\% a partir de uma amostra de conveniência. O teste de Spearman foi utilizado para avaliar a correlação entre os resultados de $\mathrm{QV}$, capacidade funcional e dosagens de NT pro-BNP.

\section{RESULTADOS}

Após a aplicação dos critérios de inclusão, 23 pacientes foram selecionados para o estudo. Três desses não concluíram o seguimento por terem apresentado fibrilação atrial ou menos de $80 \%$ de estimulação ventricular (Fig. 1).

Vinte pacientes com idade variando de 38 a 75 anos, com média de 62,7 $( \pm 9,9)$ anos, dos quais nove $(45,5 \%)$ eram do sexo feminino, completaram o protocolo do estudo (Tabela 1). A fração de ejeção média foi de $41,80 \%$ $( \pm 2,50)$. A prevalência de hipertensão arterial encontrada na amostra foi de $30 \%$ e a aplicação do teste binominal demonstrou que o uso de medicamentos anti-hipertensivos não se modificou significativamente nas diferentes fases da avaliação.

Ao acaso, 50\% dos pacientes selecionados da amostra final haviam recebido marcapasso ADAPTA ADDR03/ frequência básica 50-60 bpm. A outra metade recebeu marcapassos do modelo ENTOVIS DR-T.

A distância percorrida pelos pacientes no TV6M variou de 210 a $525 \mathrm{~m}$, com média de 390,60 ( \pm 54,73) na estimulação ventricular (VVIR). No modo DDD, variou de 375 a 650 m, com média de 396,30 ( $\pm 52,71)$. Não houve diferença significativa entre a médias da distância percorrida nos dois modos de estimulação. As médias da frequência cardíaca ao final do TC6M nos modos $\operatorname{DDD} 88,60( \pm 4,66)$ e VVIR 90,50 ( $\pm 4,57)$ não foram significativamente diferentes. A mudança de modo de estimulação não repercutiu na CF da NYHA (Tabela 2).

Os dados relativos às avaliações de $\mathrm{QV}$ pelos escores do MLHFQ revelaram piora dos aspectos considerados no domínio físico, sendo 13,35 $( \pm 3,50)$ no modo DDD e 14,60 $( \pm 3,78)$ no modo VVIR. A dimensão emocional desse questionário não sofreu influência significativa do modo de estimulação, tendo suas médias de escore variado de 8,45 $( \pm 4,10)$ DDD para 8,80 $( \pm 4,30)$ no modo VVIR, como se observa na Tabela 3.

Os resultados do AQUAREL indicaram que o modo de estimulação não repercutiu no domínio desconforto no peito, cuja média foi de $85,78( \pm 10,51)$ no modo DDD e de $84,80( \pm 10,73)$ no modo VVIR. Porém, no domínio dispneia de esforço, houve piora da média de $76.23( \pm 11,92)$ no modo DDD para 70,88 $( \pm 11,60)$ no modo VVIR. Semelhantemente, no domínio arritmia, a média obtida variou de 79,77 $( \pm 8,82)$ no modo DDD para 62,75 $( \pm 12,92)$ no modo VVIR, como demonstrado na Tabela 3. A média da dosagem de NT-proBNP enquanto os pacientes estavam sob estimulação DDD foi de 372.81 ( \pm 81.42$)$ e 495.30 ( \pm 105.4$)$ sob estimulação VVIR, como ilustra a Fig. 2.

No modo DDD, o teste de Spearman indicou correlação entre os resultados encontrados nos domínios físico e emocional do MLHFQ com seu escore total. Adicionalmente, foi possível verificar correlação entre a análise do domínio dispneia de esforço e arritmia do questionário AQUAREL, com os resultados do MLHFQ no modo DDD (Fig. 3) e no modo VVIR (Fig. 4). 


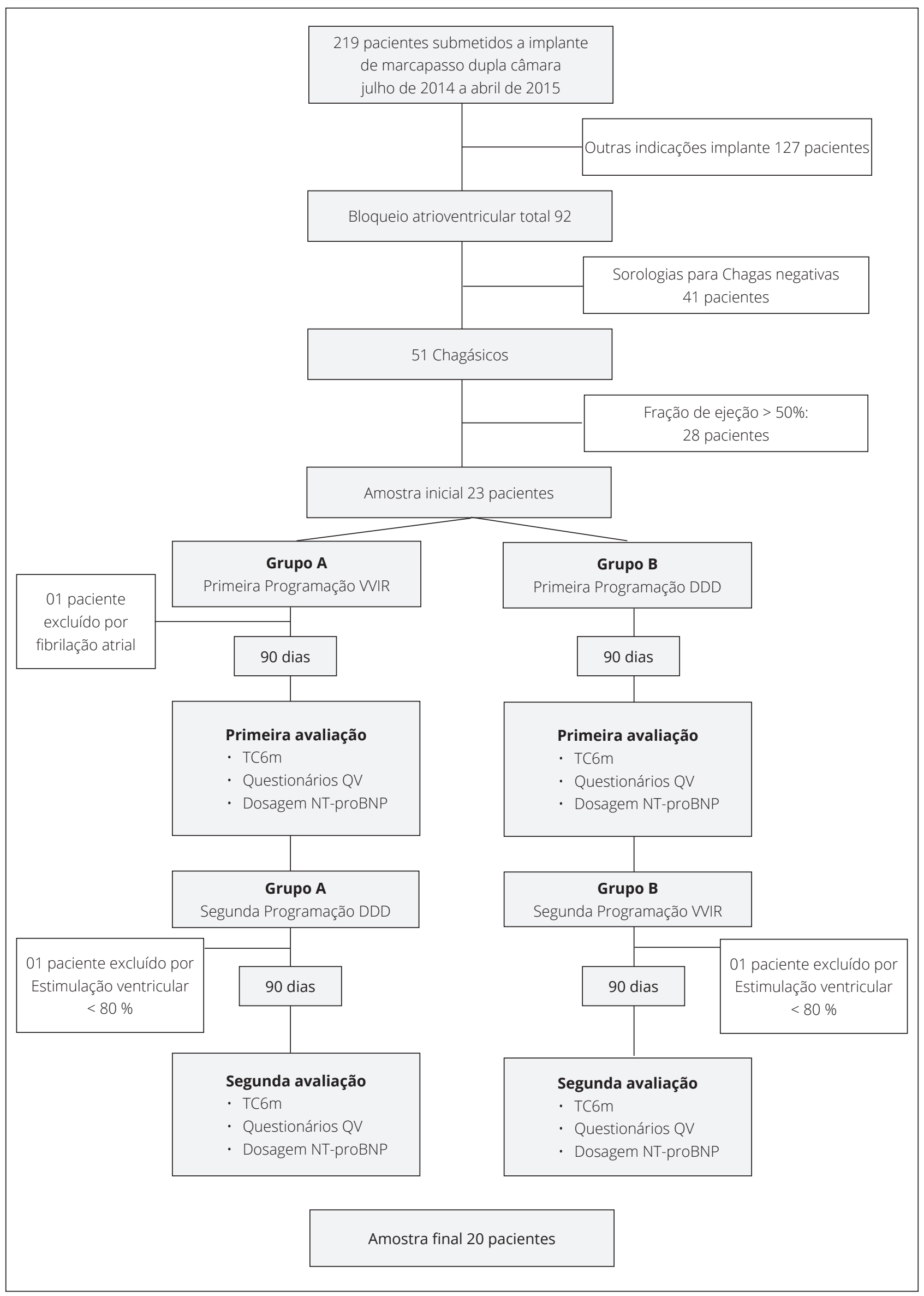

Figura 1. Delineamento do estudo. 
Tabela 1. Características dos pacientes estudados.

\begin{tabular}{|c|c|c|c|c|c|c|}
\hline Paciente & Sexo & Idade & FE Simpson (\%) & NYHA DDD & NYHA VVIR & Grupo \\
\hline 1 & $\mathrm{~F}$ & 64 & 42 & I & II & $A$ \\
\hline 2 & $M$ & 60 & 40 & II & I & $A$ \\
\hline 3 & $M$ & 60 & 45 & II & I & $A$ \\
\hline 4 & $\mathrm{~F}$ & 47 & 40 & II & II & $\mathrm{B}$ \\
\hline 5 & $\mathrm{~F}$ & 56 & 44 & 1 & I & $B$ \\
\hline 6 & $\mathrm{~F}$ & 53 & 40 & II & II & $B$ \\
\hline 7 & $\mathrm{~F}$ & 69 & 44 & III & II & $A$ \\
\hline 8 & $M$ & 38 & 44 & II & II & $B$ \\
\hline 9 & $M$ & 72 & 42 & I & ॥ & $B$ \\
\hline 10 & $M$ & 74 & 43 & I & II & $A$ \\
\hline 11 & M & 56 & 40 & II & II & $B$ \\
\hline 12 & M & 56 & 40 & II & II & A \\
\hline 13 & $\mathrm{~F}$ & 75 & 46 & II & III & B \\
\hline 14 & $\mathrm{~F}$ & 71 & 46 & II & II & B \\
\hline 15 & $M$ & 59 & 40 & II & II & $A$ \\
\hline 16 & $M$ & 75 & 39 & III & II & B \\
\hline 17 & $M$ & 72 & 43 & II & II & $B$ \\
\hline 18 & $M$ & 62 & 42 & II & II & $A$ \\
\hline 19 & $\mathrm{~F}$ & 69 & 38 & II & II & A \\
\hline 20 & $\mathrm{~F}$ & 65 & 38 & II & II & $A$ \\
\hline
\end{tabular}

FE Simpson (\%) = Fração de Ejeção do ventrículo esquerdo; NYHA DDD = Classe Funcional (CF) da New York Heart Association após 90 dias de estimulação sob o modo atrioventricular; NYHA WVIR = CF da New York Heart Association após 90 dias de estimulação sob o modo de estimulação ventricular com resposta de frequência determinada por sensor e inibição por evento elétrico ventricular sentido pelo marcapasso.

Tabela 2. Resultado do teste do qui-quadrado post hoc comparando as frequências das classificações da New York Heart Association (NYHA) entre o DDD e VVIR.

\begin{tabular}{cccc}
\hline \multirow{2}{*}{ Classificação NYHA } & \multicolumn{2}{c}{ Grupo $\mathbf{n}(\%)$} & p-valor* \\
\cline { 2 - 4 } & DDD & VVIR & 0,68 \\
I & $4(20,0)$ & $3(15,0)$ & 0,47 \\
III & $14(70,0)$ & $16(80,0)$ & 0,55 \\
\hline
\end{tabular}

$\mathrm{DDD}=$; NYHA = classe funcional da New York Heart Association; VVIR = modo de estimulação ventricular com resposta de frequência determinada por sensor e inibição por evento elétrico ventricular sentido pelo marcapasso; *Qui-quadrado post hoc.

Tabela 3. Resultado da comparação dos domínios do Minnesota Living with Heart Failure Questionnaire (MLHFQ) entre os modos DDD e VIR.

\begin{tabular}{lccc}
\hline \multirow{2}{*}{ Minnesota Living with Heart Failure Questionnaire } & \multicolumn{2}{c}{ Média \pm desvio padrão } & \multirow{2}{*}{ p-valor* } \\
\cline { 2 - 4 } & DDD & VVIR & 0,51 \\
\hline Emocional & $8,45 \pm 4,10$ & $14,60 \pm 4.30$ & 0,03 \\
Físico & $13,35 \pm 3,50$ & $33,15 \pm 5.89$ & 0,73 \\
Total & $33,75 \pm 7,30$ & VVIR & p-valor* \\
\hline Assesment of QUAlity of life and RELated events & DDD & $84,80 \pm 10,73$ & 0,14 \\
\hline Desconforto no peito & $85,78 \pm 10,51$ & $70,88 \pm 11,60$ & 0,005 \\
Dispneia de esforço & $76,23 \pm 11,92$ & $62,75 \pm 12,92$ & $<0,001$ \\
Arritmia & $79,77 \pm 8,82$ & &
\end{tabular}

* Teste de Wilcoxon. 


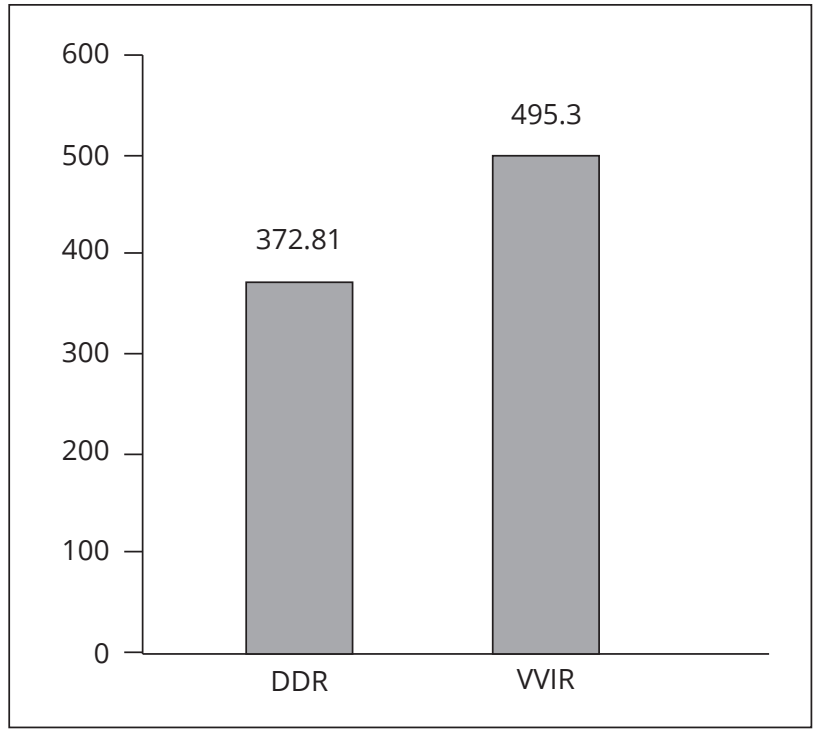

Figura 2. Níveis séricos da fração $\mathrm{N}$-terminal do peptídeo natriurético cerebral ( $N$-terminal pro b-type natriuretic peptide) nos modos DDD e VIR (intervalo de confiança de 95\%).

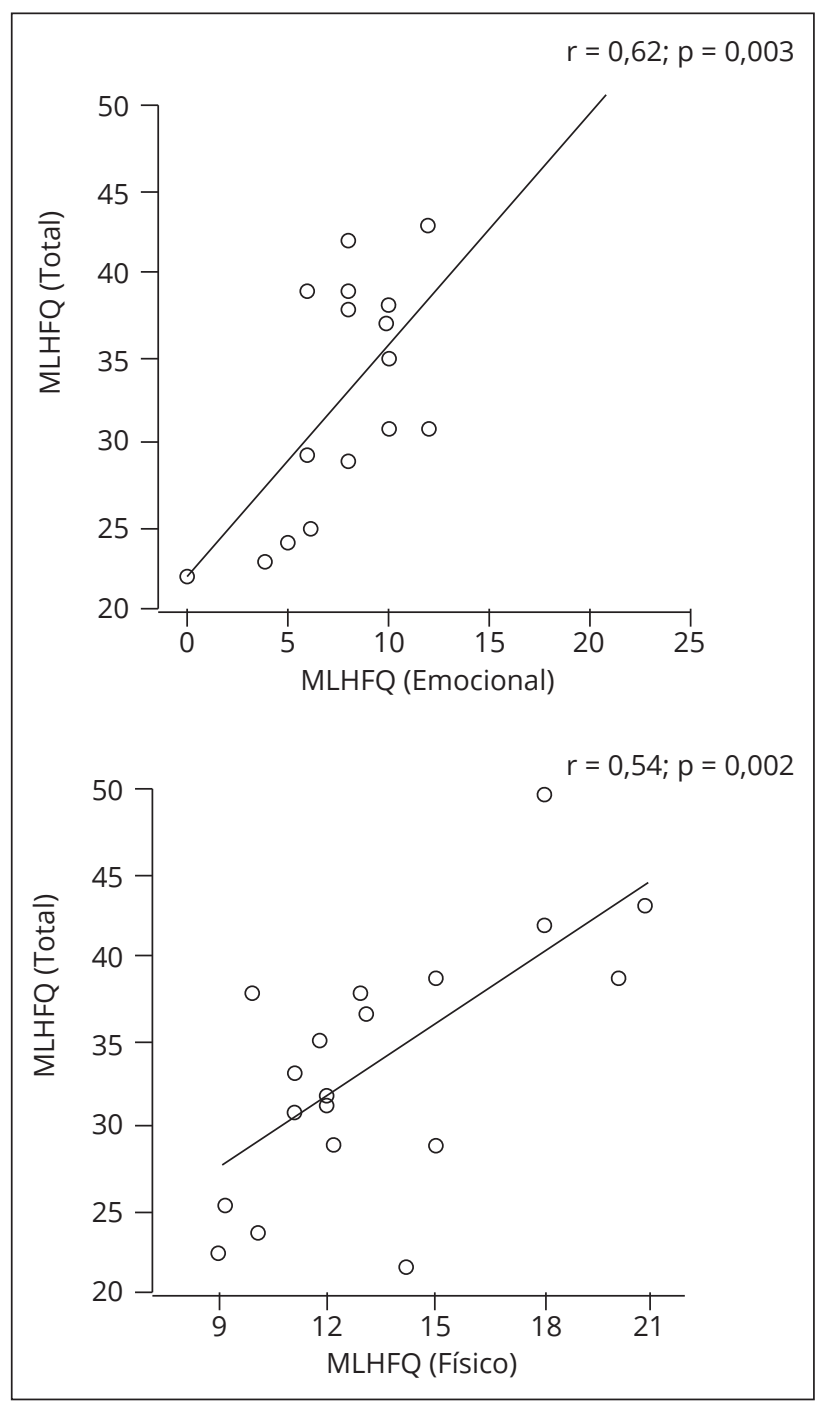

Figura 3. Gráfico de dispersão demonstrando os resultados da correlação de Spearman entre as variáveis de qualidade de vida no modo DDD.

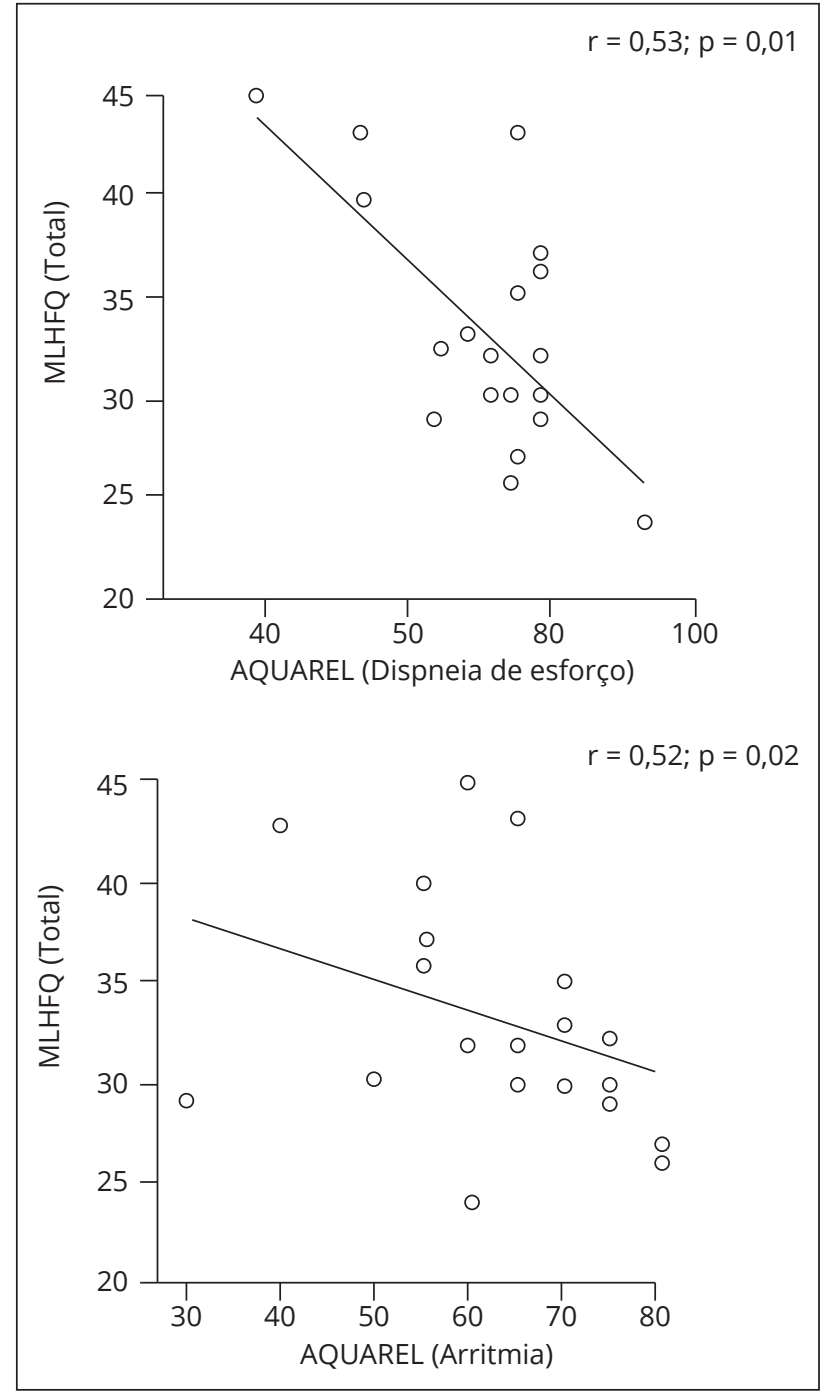

Figura 4. Gráfico de dispersão demonstrando os resultados da correlação de Spearman entre variáveis da qualidade de vida no modo no modo VVIR.

\section{DISCUSSÃO}

Os resultados deste trabalho demonstram que o modo de estimulação $\mathrm{AV}$ proporciona melhor impacto na $\mathrm{QV}$ de acordo com ambos os instrumentos utilizados.

Ainda que sem efeito significativo no escore global do MLHFQ, ao analisar-se seu componente físico, percebemos que a estimulação DDD apresentou resultados superiores $(\mathrm{p}=0,03)$. O MLHFQé um instrumento com acurácia bem definida para pacientes com disfunção ventricular ${ }^{10}$, incluindo a miocardiopatia chagásica ${ }^{8}$. Esse questionário foi também empregado para detectar repercussões da estimulação artificial na QV, como no estudo de Santos et al. ${ }^{11}$, no qual analisaram a influência da atividade do sensor em pacientes sob modo de estimulação DDD. 
As respostas obtidas pelo AQUAREL, que englobam os domínios dispneia de esforço $(p=0,005)$ e arritmia ( $\mathrm{p}<0,001)$, evidenciaram melhor percepção de QV sob o modo de estimulação DDD. $\mathrm{Na}$ amostra deste estudo, as piores pontuações foram detectadas no domínio arritmia. Esse achado é concordante com o estudo de Oliveira et al. ${ }^{12}$, no qual médias baixas no questionário AQUAREL foram características dos pacientes chagásicos, além de estarem associadas a piores estágios de insuficiência cardíaca, segundo a CF. Em contrapartida, Barros et al. ${ }^{13}$ mensuraram a QV de uma população de idosos sem DCh com aplicação do AQUAREL e observaram que as piores medidas se concentravam no domínio dispneia de esforço. Essas considerações realçam o papel da manifestação arritmogênica característica da DCh e a magnitude de seu reflexo na percepção da $\mathrm{QV}$ desses indivíduos.

Nota-se, portanto, a importância do emprego de instrumentos específicos para avaliação da $\mathrm{QV}$ em chagásicos sob estimulação artificial. A utilização do SF-36, instrumento de avaliação global, não detectou diferenças na $\mathrm{QV}$ sob efeitos da estimulação VVIR ou DDD/R em pacientes idosos de um ensaio clínico randomizado ${ }^{14}$. Teno et al. ${ }^{15}$ selecionaram pacientes chagásicos na ocasião da troca do gerador do marcapasso e concluíram não haver diferenças na $Q V$ entre os modos DDD ou VVIR segundo o SF-36. É importante a utilização de instrumentos globais e específicos para avaliação das intervenções em saúde. Há evidências de que os valores dos escores do SF-36 se modificam significativamente nos primeiros meses após o implante do marcapasso, enquanto os resultados do AQUAREL podem ser percebidos ao longo do seguimento de cinco anos ${ }^{16}$.

$\mathrm{Na}$ casuística deste estudo, apesar de a avaliação da capacidade funcional pelo TC6M não ter demonstrado que a estimulação $\mathrm{AV}$ proporciona melhor desempenho do que a unicameral $(p=0,16)$, seus valores correlacionaram-se com as avaliações de QV tanto pelo MLHFQ $(r=0,62$; $\mathrm{p}=0,003)$ quanto pelo AQUAREL $(\mathrm{r}=0,44 ; \mathrm{p}=0,04)$ no modo VVIR. Com esses resultados, verificou-se que os pacientes com pior capacidade funcional também apresentavam piores escores de QV.

Em um estudo duplo-cego randomizado com participantes não chagásicos ${ }^{17}$, não se encontrou alteração na capacidade funcional pelo TC6M com a mudança dos modos de estimulação. Sá et al. ${ }^{18}$ verificaram que, após oito meses de implante de marcapasso, houve piora dos resultados obtidos no TC6M em relação ao primeiro mês após o implante. Portanto, apesar de o TC6M ser considerado um teste submáximo desencadeador de respostas globais e integradas dos sistemas envolvidos durante o exercício, não é capaz de evidenciar diferenças relacionadas aos modos de estimulação ventricular ou $\mathrm{AV}^{15}$.

A dosagem dos peptídeos natriuréticos para detectar precocemente a progressão da disfunção ventricular tem ganhado destaque nos últimos anos ${ }^{19}$. A estimulação ventricular pode causar elevação precoce dos níveis NT-proBNP de forma independente de alteração ecocardiográfica ${ }^{20}$. No estudo de Souza et al. ${ }^{21}$, realizou-se a dosagem de peptídeo natriurético cerebral (brain natriuretic peptide - BNP) no sexto mês e um ano após o implante do marcapasso. Esses autores encontraram incremento significativo do BNP na segunda dosagem, quando a fração de ejeção do ventrículo esquerdo não mostrava diferença em relação à medida inicial ${ }^{21}$.

Os critérios de seleção utilizados nesse estudo permitiram a formação de uma amostra homogênea com disfunção ventricular ao implante do marcapasso. Verificou-se que o modo de estimulação VVIR concorreu em elevação significativa dos níveis de NT-proBNP. Essa elevação pode estar relacionada à dissincronia $\mathrm{AV}$ ou à piora da regurgitação mitral secundária à estimulação apical direita. Não foi discriminado, na análise deste estudo, o sítio de estimulação ventricular, porém sabe-se que seus efeitos a curto prazo não estão completamente elucidados ${ }^{22}$.

A única variável com a qual os elevados níveis de NT-proBNP se correlacionaram na amostra deste estudo foi a distância percorrida no TC6M no modo VVIR, sugerindo a íntima relação da capacidade funcional com a elevação sérica desse peptídeo.

Esses dados se referem a um seguimento de 90 dias, em uma amostra constituída de 20 pacientes. Isso torna a observação não conclusiva, mas capaz de dar informações importantes acerca do tipo de estimulação cardíaca no paciente com cardiomiopatia chagásica.

\section{CONCLUSÃO}

O modo DDD proporciona melhor QV a pacientes chagásicos com disfunção ventricular sem repercutir em sua capacidade funcional medida pelo TC6M. O modo de estimulação VVIR resultou em elevação dos níveis séricos de NT-proBNP após três meses de seguimento. 


\section{CONTRIBUIÇÃO DOS AUTORES}

Conceitualização, Santana DR e Rassi S; Metodologia, Santana DR; Lima AMC e Rassi S; Investigação, Santana

DR; Santana Filho GP e Rocha ZB; Redação - Primeira versão, Santana DR e Rassi S; Redação - Revisão \& Edição, Santana DR; Rassi S; Nery MW e Gardenghi G; Recursos, Santana DR; Supervisão, Rassi S.

\section{REFERÊNCIAS}

1. Rassi AJ, Rassi A, Marin-Neto J. Chagas disease. Lancet. 2010;375(9723):1388-402. https://doi.org/10.1016/S01406736(10)60061-X

2. World Health Organization. Chagas disease in Latin America: an epidemiological update based on 2010 estimates. Weekly Epidemiol Rec. 2015;90(6):33-43.

3. Sabino EC, Ribeiro AL, Salemi VMC, Di Lorenzo Oliveira C, Antunes AP, Menezes MM, et al. Ten-year incidence of Chagas cardiomyopathy among asymptomatic Trypanosoma cruzi seropositive former blood donors. Circulation. 2013;127(10):1105-15. https://doi.org/10.1161/ circulationaha.112.123612

4. Pachon JC, Pereira W, Batista Junior WD, Pachón JPM, Pachón CEI, Vargas RNA, et al. RBM - Registro Brasileiro de Marcapassos, Ressincronizadores e Desfibriladores. Relampa. 2013;26(1):39-49.

5. Rassi A, Rassi Jr A, Faria GHDC. História natural do bloqueio atrioventricular total de etiologia chagásica. Arq Bras Cardiol. 1992;59(Suppl II):191.

6. Martinelli Filho M, Zimerman LI. Diretrizes brasileiras de dispositivos cardíacos eletrônicos implantáveis (DCEI). Arq Bras Cardiol. 2007;89(6):e210-38.

7. American Thoracic Association. ATS statement: guidelines for the six-minute walking test. Am J Resp Critical Care Med. 2002;166(1):111-7. https://doi.org/10.1164/ ajrccm.166.1.at1102

8. Carvalho VO, Guimarães GV, Carrara D, Bacal F, Bocchi EA. Validação da versão em português do Minnesota Living with Heart Failure Questionnaire. Arq Bras Cardiol. 2009;93:3944. https://doi.org/ 10.1590/S0066-782X2009000700008

9. Beasley TM, Schumacker RE. Multiple regression approach to analyzing contingency tables: post hoc and planned comparison procedures. J Exp Educ. 1995;64(1):79-93. https://doi.org/10.1080/00220973.1995.9943797

10. Rector TS, Kubo SH, Cohn JN. Validity of the Minnesota Living with Heart Failure Questionnaire as a measure of therapeutic response to enalapril or placebo. Am J Cardiol. 1993;71(12):1106-7.

11. Santos JRC. Marcapassos dupla-câmara com resposta de frequência melhoram a qualidade de vida dos pacientes? Relampa. 2002;15(4):201-5.

12. Oliveira BG, Abreu MNS, Abreu CDG, Rocha MOC, Ribeiro AL. Health-related quality of life in patients with Chagas disease. Rev Soc Bras Med Trop. 2011;44:150-6. https://doi. org/10.1590/S0037-86822011005000002

13. Barros RTD, Carvalho SMRD, Silva MAM, Borges JBC. Avaliação de aspectos da qualidade de vida em pacientes pós-implante de marcapasso cardíaco. Rev Bras Cir
Cardiovasc. 2014:37-44. https://doi.org/10.5935/16789741.20140009

14. Lamas GA, Orav EJ, Stambler BS, Ellenbogen KA, Sgarbossa EB, Huang SKS, et al. Quality of life and clinical outcomes in elderly patients treated with ventricular pacing as compared with dual-chamber pacing. NEJM. 1998;338(16):1097-104. https://doi.org/10.1056/NEJM199804163381602

15. Teno LAC, Costa R, Martinelli Filho M, Castilho FCT, Ruiz I. Mudança do modo WI para DDD ("upgrade") na troca eletiva do gerador de pulsos: análise comparativa clínicofuncional. Arq Bras Cardiol. 2007;88(2):128-33. https://doi. org/10.1590/S0066-782X2007000200001

16. Udo EO, van Hemel NM, Zuithoff NP, Nijboer $H$, Taks W, Doevendans PA, et al. Long term quality-of-life in patients with bradycardia pacemaker implantation. Inter J Cardiol. 2013;168(3):2159-63. https://doi.org/10.1016/j. ijcard.2013.01.253

17. Ouali S, Neffeti E, Ghoul K, Hammas S, Kacem S, Gribaa R, et al. DDD versus WIR pacing in patients, ages 70 and over, with complete heart block. Pacing Clin Electrophysiol. 2010;33(5):5839. https://doi.org/10.1111/j.1540-8159.2009.02636.x

18. Sá LAB, Rassi S, Batista MAL. Efeitos da estimulação ventricular convencional em pacientes com função ventricular normal. Arq Bras Cardiol. 2009;93:167-73. https://doi.org/10.1590/S0066-782X2009000800016

19. Porapakkham P, Porapakkham P, Zimmet H, Billah B, Krum H. B-type natriuretic peptide-guided heart failure therapy: a meta-analysis. Arch Intern Med. 2010;170(6):507-14. https:// doi.org/10.1001/archinternmed.2010.35

20. Naegeli B, Kurz DJ, Koller D, Straumann E, Furrer M, Maurer $D$, et al. Single-chamber ventricular pacing increases markers of left ventricular dysfunction compared with dual-chamber pacing. Europace. 2007;9(3):194-9. https://doi.org/10.1093/ europace/eul186

21. Souza SF, Nascimento B, Nunes MCP, da Silva JLP, Carvalho $V T$, Beaton $A Z$, et al. Effect of pacemaker site on B-type natriuretic peptide levels, and left ventricular function in a population with high prevalence of Chagas disease. Inter J Cardiol. 2015;190:315-8. https://doi.org/10.1016/j. ijcard.2015.04.127

22. Nikoo MH, Ghaedian MM, Kafi M, Fakhrpour A, Jorat MV, Pakfetrat M, et al. Effects of right ventricular septal versus apical pacing on plasma natriuretic peptide levels. J Cardiovasc Dis Res. 2011;2(2):104-9. https://doi. org/10.4103\%2F0975-3583.83036 